\title{
Time-dependently expressed markers and the characterization for premature senescence induced by ionizing radiation in MCF7
}

\author{
BONG CHO KIM ${ }^{1}$, NA-KYUNG HAN ${ }^{1}$, HAE-OK BYUN $^{1}$, SEUNG-SOO KIM $^{2}$, EUN-KYUNG AHN ${ }^{3}$, \\ IN-SUN CHU ${ }^{4}$, SUN-HEE LEEM ${ }^{3}$, CHEOL-KOO LEE $^{2}$ and JAE-SEON LEE ${ }^{1}$ \\ ${ }^{1}$ Division of Radiation Cancer Research, Korea Institute of Radiological and Medical Sciences, Seoul 139-706; \\ ${ }^{2}$ Division of Biotechnology, College of Life Sciences and Biotechnology, Korea University, Seoul 136-701; \\ ${ }^{3}$ Department of Biological Science, Dong-A University, Busan 604-714; ${ }^{4}$ Korean BioInformation Center, \\ Korea Research Institute of Bioscience and Biotechnology, Daejeon 305-333, Korea
}

Received March 3, 2010; Accepted April 26, 2010

DOI: $10.3892 /$ or_00000872

\begin{abstract}
Senescence has been suggested as a defense mechanism to block sporadic induction of cancer cells. Radiation treatment induces proliferating cancer cells to turn into non-proliferating senescent cells in vitro. To characterize transcriptional reprogramming after radiation treatment, we measured the gene expression profiles of MCF7 at different time points after treatment. In these experiments, we found that IR induced premature senescence in MCF7 cells, and IR treatment resulted in significant changes in the expression of 305 marker genes ( $<1 \%$ FDR), which were strongly correlated $(|r|>0.9)$ with IR treatment in a time-dependent manner. Functional analysis of these markers indicated that the dynamics of cytoskeletal structure and lysosomal activity gradually increased. The expression of maker genes for modulator proteins, that were responsible for the dynamics of actin stress fibers and focal adhesion, displayed a particularly strong positive correlation with senescenceassociated (SA) morphological changes through time. We also observed a strong induction of genes related to lysosomal metabolic activity, which was accompanied by an increase in the number of SA-B-Gal positive cells. However, the expression of genes for cell cycle progression, post-transcription and translation activities gradually decreased after radiation treatment. Especially, we observed clear cell cycle arrest specifically at the S and G2/M phases
\end{abstract}

Correspondence to: Dr Jae-Seon Lee, Division of Radiation Cancer Research, Korea Institute of Radiological and Medical Sciences, Seoul 139-706, Korea

E-mail: jaeslee@kcch.re.kr

Dr Cheol-Koo Lee, Division of Biotechnology, College of Life Sciences and Biotechnology, Korea University, Anam-dong, Seongbuk-gu, Seoul 136-701, Korea

E-mail: cklee2005@korea.ac.kr

Key words: ionizing radiation, premature senescence, MCF7, gene expression profile with consistent down-regulation of genes for microtubule assembly/disassembly or spindle biogenesis.

\section{Introduction}

Radiotherapy is one of the best therapeutic strategies for cancer treatment. The cellular response to ionizing radiation (IR) varies and encompasses a spectrum ranging from growth arrest to apoptotic cell death (1). To obtain a better understanding of IR-induced molecular processes, IR response genes have been identified and shown to belong to the DNA damage response, regulation of cell cycle and cell proliferation, apoptosis, and intracellular signaling cascade (2). However, in evaluating the relative contribution of apoptosis and senescence to tumor regression, recent data suggest that senescence may play a more significant role in the primary mechanism underlying loss of the self-renewal capacity in IR- or drug-treated cancer cells (3-5).

Cellular senescence is a stress-response phenomenon in which cells lose the ability to proliferate. It is induced by telomere shortening, dysregulation of oncogenes or tumor suppressor genes, or exposure to a sub-lethal dose of DNA damaging agents or oxidative stresses $(6,7)$. Senescent cells undergo functional and morphological changes including large and flattened cell shape, senescence-associated Bgalactosidase (SA- $\beta-$ Gal) activity, and senescence-associated heterochromatic foci (SAHF) formation $(6,8,9)$. Recent studies suggest that one of the critical steps of carcinogenesis is an escape from senescence in preneoplastic lesions. For this reason, senescence is considered as a cellular defense mechanism to prevent tumorigenesis, and induction of premature senescence in immortalized cancer cells is a promising treatment for tumors (10-12). However, the mechanism underlying cancer cell senescence induced by the treatment with IR or drugs is not fully understood.

Cells undergoing stress-induced premature senescence remain metabolically active and display characteristic changes in gene expression patterns, as well as the aforementioned senescence-specific features (6). DNA microarray analysis has been used to assess alterations in gene expression under a range of different cellular conditions. 
The advent of gene expression profiling and bioinformatics analysis greatly facilitated the identification of genes and pathways that regulate cellular senescence. Most of these changes, such as activation of p53 or the up-regulation of the cyclin-dependent kinase (CDK) inhibitors, relate directly to the establishment and maintenance of growth arrest. Also, it is worth noting that senescent cells produce increased amounts of various proteins, which are involved in cell cycle regulation, immune and inflammation, cytoskeleton, stress response, and metabolism (13-15). Recently, the data obtained from several gene expression profiles and supporting functional data have lead to the identification of the key genes regulating senescence and immortalization, relating to the following 6 pathways (16): the cell cycle $\mathrm{pRB} / \mathrm{p} 53$, cytoskeletal, interferon-related, insulin-like growth factor (IGF)-related, mitogen-activated protein kinase (MAPK), and oxidative stress pathways. Furthermore, there is increasing evidence that secreted proteins from senescent cells are primarily responsible for altering the tissue microenvironment surrounding tumors, and can affect neighboring senescent and preneoplastic cells through paracrine signaling $(17,18)$. However, although IR is one of the most potent inducers of senescence, the mechanism by which IR induces premature senescence in cancer cells is still largely unknown.

In the present study, we categorized differentially expressed genes during induction of premature senescence in breast cancer cells by IR, and scrutinized the biological processes of stress-induced senescence. The present data could help provide a better understanding of IR-induced premature senescence in cancer cells, and aid in the development of novel strategies for diagnostic and therapeutic purposes in cancer treatment.

\section{Materials and methods}

Cell culture and IR exposure. Human breast cancer MCF7 cells were purchased from the American Type Culture Collection and cultured in DMEM (MyClone, Logan, UT) supplemented with 10\% FBS (WelGENE, Daegu, Korea), and $1 \%$ penicillin/streptomycin solution at $37^{\circ} \mathrm{C}$ in a $5 \%$ $\mathrm{CO}_{2}$ incubator. For irradiation, cells were exposed to $\gamma$-ray with a ${ }^{137} \mathrm{Cs} \gamma$-ray source (Atomic Energy of Canada, Mississauga, Canada) at a dose rate of $3.0 \mathrm{~Gy} / \mathrm{min}$.

$S A-\beta-G a l$ assay. To determine the senescent phenotype, cells were stained for $\beta$-galactosidase activity described in Dimri et al (8) with minor modifications. Briefly, cells were washed in PBS, fixed at room temperature for 3-5 min in $3 \%$ formaldehyde $/ 0.2 \%$ glutaraldehyde, washed with PBS, and incubated at $37^{\circ} \mathrm{C}$ (without $\mathrm{CO}_{2}$ ) with a fresh staining solution consisting of $1 \mathrm{mg} / \mathrm{ml}$ 5-bromo-4-chloro-3-indolyl ß-D-galactoside (X-Gal, from a stock solution of $20 \mathrm{mg} / \mathrm{ml}$ in dimethylformamide) in $40 \mathrm{mM}$ citric acid/sodium phosphate, $\mathrm{pH} 6.0,5 \mathrm{mM}$ potassium ferrocyanide, $5 \mathrm{mM}$ potassium ferricyanide, $150 \mathrm{mM} \mathrm{NaCl}$ and $2 \mathrm{mM} \mathrm{MgCl}_{2}$. Staining was evident in $4-8 \mathrm{~h}$ and maximal at $12-16 \mathrm{~h}$.

Colony forming assay (CFA). Clonogenicity was examined using a colony forming assay. Cells $\left(1 \times 10^{3}\right)$ were seeded onto $35-\mathrm{mm}$ plates in culture media containing $10 \%$ FBS for $7-10$ days. Colonies were stained with Diff Quick (Sysmex Corp., Kobe, Japan) and counted using an Artek Counter (Imaging Products International Inc., Chantilly, VA).

FACS analysis. To determine cell cycle distribution, cells were collected by trypsinization, fixed in $70 \%$ ethanol, washed in PBS, and resuspended in $1 \mathrm{ml}$ of PBS containing $1 \mathrm{mg} / \mathrm{ml} \mathrm{RNase}$ and $50 \mu \mathrm{g} / \mathrm{ml}$ propidium iodide. After incubation in the dark for $30 \mathrm{~min}$ at room temperature, cell cycle distributions were analyzed using an EPICS flow cytometer (Beckman-Coulter Inc., Miami, FL, USA). The data were analyzed using Multicycle software (Phoenix Flow Systems, San Diego, CA, USA).

Western blot analysis. Cells were washed with ice-cold phosphate-buffered saline (PBS), lysed in $50 \mu \mathrm{l}$ of ice-cold RIPA buffer (25 mM Tris- $\mathrm{HCl}, \mathrm{pH} 7.4,150 \mathrm{mM} \mathrm{NaCl}$, 5 mM EDTA, $1 \%$ NP-40, $0.5 \%$ sodium deoxycholate, $0.5 \%$ SDS, $1 \mathrm{mM} \mathrm{Na}_{3} \mathrm{VO}_{4}, 5 \mathrm{mM} \mathrm{NaF}$, and $1 \mathrm{mM}$ phenylmethylsulfonyl fluoride), and collected by scraping with a rubber policeman. Cell disruption was achieved by vortexing repeatedly at $30-$ sec intervals on ice. Particulate debris was removed by centrifugation at $13,600 \mathrm{x} g$ for $10 \mathrm{~min}$. The protein concentration in the supernatants was determined using the BCA method. Equal amounts of proteins $(30 \mu \mathrm{g})$ were separated on 10-12\% SDS-polyacrylamide gels and then transferred onto nitrocellulose membranes. The membranes were incubated overnight at $4^{\circ} \mathrm{C}$ with one of the specific antibodies. After washing three times in TBST, horseradish peroxidase-conjugated goat anti-mouse, goat anti-rabbit, or donkey anti-goat antibodies were applied. The proteins were visualized using enhanced chemiluminescence with a LAS-3000 image system (Fujifilm Medical systems USA Inc., Stanford, USA).

Sample labeling and Illumina BeadChip array hybridization. Total RNA from MCF7 cells was isolated using the TRIzol reagent (Invitrogen, CA) according to the manufacturer's instruction. The quality of total RNA was checked by gel electrophoresis and RNA concentrations were determined using the Ultrospec 3100 pro spectrophotometer (Amersham Bioscience, Buckinghamshire, UK). Biotin-labelled cRNA samples for hybridization were prepared according to the Illumina recommended sample labeling procedure. Briefly, $500 \mathrm{ng}$ of total RNA was used for cDNA synthesis, followed by an amplication/labeling step (in vitro transcription) to synthesize biotin-labelled cRNA according to the Illumina ${ }^{\circledR}$ TotalPrep RNA Amplification kit (Ambion Inc., Austin, TX). The cRNA concentrations were measured by the RiboGreen method (Quant-iT ${ }^{\mathrm{TM}}$ RiboGreen ${ }^{\circledR}$ RNA assay kit; InvitrogenMolecular Probes, ON, Canada) using the Victor3 spectrophotometer (Perkin-Elmer, CT) and cRNA quality was checked on a $1 \%$ agarose gel. Labeled, amplified cRNA (1.5 $\mu \mathrm{g}$ per array) was hybridized to a ver. 2 of the Illumina Human-6 BeadChip (48 K) according to the manufacturer's instructions (Illumina, Inc., San Diego, CA). Array signals were developed by Amersham fluorolink streptavidin-Cy3 (GE Healthcare Bio-Sciences, Little Chalfont, UK) following the protocol outlined in the BeadChip manual. Arrays were scanned with an Illumina Bead array Reader confocal scanner 
A.

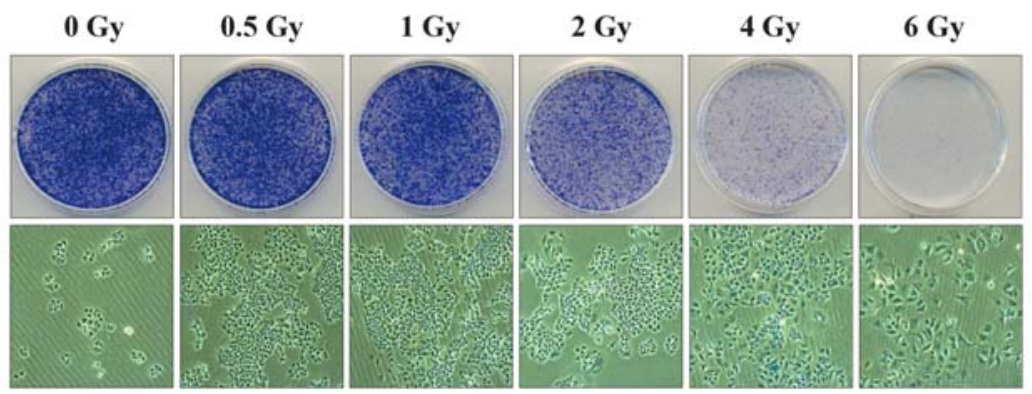

B.
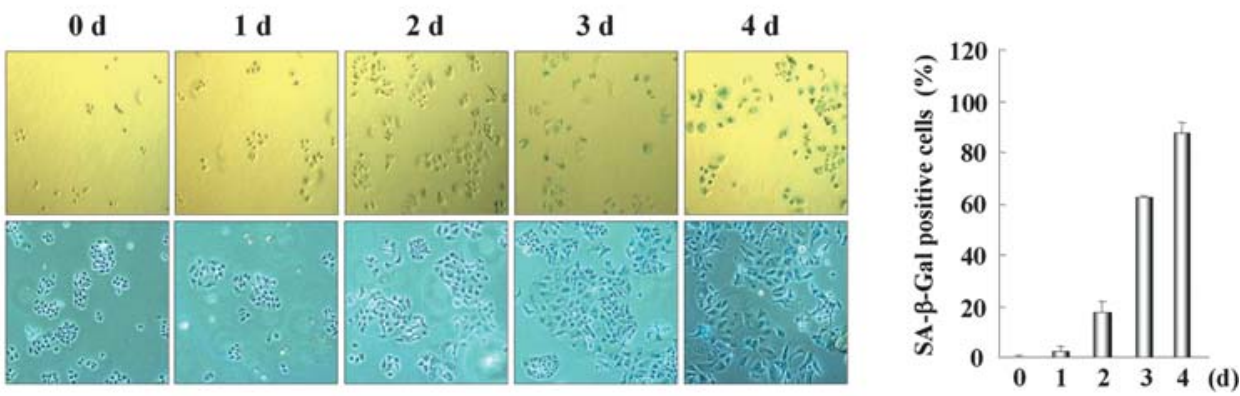

C.

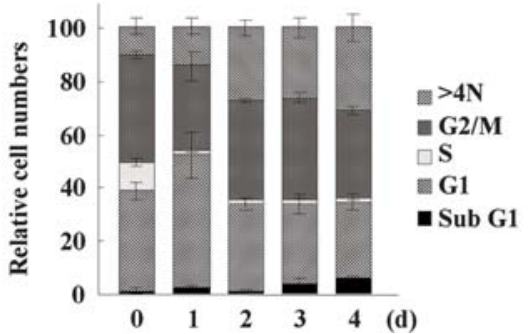

D.

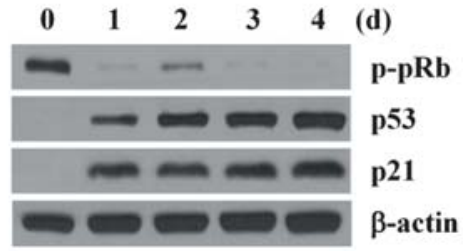

Figure 1. Induction of premature senescence in MCF7 human breast cancer cells by exposure to IR. (A) Colony forming assay and cellular morphology. After exposure to various doses of IR, colony forming abilities were analyzed on the 7th day (d) and cellular morphologies were observed on the 4th day. (B) Cellular morphology and SA-B-Gal activity in 6 Gy of IR exposed MCF7 cells. Cell images and SA-B-Gal activity were acquired during 4 days after IR exposure. Percentages of SA-B-Gal positive cells are shown in the graph. (C) Cell cycle distribution of IR-exposed cells. Irradiated MCF7 cells were stained with propidium iodide and sub-G1, G1, S, G2/M, and aneuploid populations were quantified by FACS. (D) Western blot analysis in 6 Gy of IRexposed cells. The cell cycle regulatory proteins were examined with their specific antibodies by immunoblot.

(BeadStation 500GXDW; Illumina, Inc.). Array hybridizations were conducted with four sets of RNA samples from independently cultured cell samples per unit time. Array data processing and analysis was performed using Illumina BeadStudio software.

Transcriptome data analysis. The raw signals were transformed into $\log _{2}$ scale. The quantile normalized dataset was analyzed by ANOVA as a function of time. ANOVA p-values were used to evaluate for the false positive proportion by false discovery rate analysis (Partek Genomics Suite 6.3). The Pearson correlation coefficients were computed to quantify the relation between expression and time. The data discussed in this publication have been deposited in the NCBI's Gene Expression Omnibus and are accessible through GEO Series accession number GSE21748.

\section{Results}

IR induces premature senescence in MCF7 cells. To determine the efficiency with which IR induces premature sene- scence in cancer cells, we first examined the morphological changes and clonogenicities in MCF7 human breast cancer cells exposed to various doses of IR. Colony forming ability was markedly decreased by IR irradiation, and senescencespecific morphologies, such as a large and flat cellular shape, progressively increased in a dose-dependent manner (Fig. 1A). We found that the 6 Gy of the IR dose effectively induced premature senescence. To confirm the effect of 6 Gy IR on senescence induction, cellular morphology and SA- $\beta-G a l$ activity were examined for 4 days. The number of cells that displayed a senescence-specific morphology and positive SA-ß-Gal activity gradually increased up to 4 days (Fig. 1B). In addition, cell proliferation was greatly retarded due to senescence induction, and almost all cells were positively stained with SA-ß-Gal after 4 days. When we analyzed the cell cycle distribution by flow cytometry, growth arrest in the $\mathrm{G} 1 / \mathrm{S}$ phase was observed and the subG1 population was $<10 \%$ up to 4 days (Fig. 1C). Molecular changes related with cell cycle arrest were observed in IR-exposed cells. Loss of phospho-pRB, accumulation of p53, and induction of p21 were detected 1 day after irradiation and these changes were 
Table I. List of genes responsible for the regulation of actin polymerization/depolymerization.

\begin{tabular}{llccll}
\hline Illumina ID & Symbol & FDR & $r$ & Role & Gene name \\
\hline ILMN_15394 & SVIL & $4.70 \mathrm{E}-09$ & 0.98 & Linker & Supervillin \\
ILMN_5918 & PALLD & $2.80 \mathrm{E}-06$ & 0.96 & Colocalizer & Palladin, cytoskeletal associated protein \\
ILMN_17386 & TRIP6 & $1.69 \mathrm{E}-06$ & 0.95 & Colocalizer & Thyroid hormone receptor interactor 6 \\
ILMN_18161 & RND1 & $1.87 \mathrm{E}-06$ & 0.93 & Modulator & Rho family GTPase 1 \\
ILMN_16821 & SHROOM3 & 6.84E-08 & 0.93 & Colocalizer & Shroom family member 3 \\
ILMN_2137 & ZYX & 2.26E-05 & 0.93 & Linker & Zyxin \\
ILMN_19658 & RHOU & 2.37E-05 & 0.93 & Modulator & Ras homolog gene family, member U \\
ILMN_6304 & FGD3 & $9.75 \mathrm{E}-07$ & 0.92 & Modulator & FYVE, RhoGEF and PH domain containing 3 \\
ILMN_2265 & RHOQ & $1.50 \mathrm{E}-04$ & 0.92 & Modulator & Ras homolog gene family, member Q \\
ILMN_9535 & WASF3 & 5.65E-06 & 0.92 & Linker & WAS protein family, member 3 \\
ILMN_752 & TMSB4X & 2.39E-06 & 0.91 & Modulator & Thymosin B 4, X-linked \\
ILMN_137841 & GSN & $4.00 \mathrm{E}-06$ & 0.91 & Modulator & Gelsolin (amyloidosis, Finnish type) \\
ILMN_27553 & RHOC & $1.30 \mathrm{E}-06$ & 0.90 & Linker & Ras homolog gene family, member C \\
ILMN_22028 & ARHGAP19 & $5.20 \mathrm{E}-08$ & -0.90 & Modulator & Rho GTPase activating protein 19 \\
\hline
\end{tabular}

sustained for up to 4 days (Fig. 1D). Interestingly, the transcript of p21 was significantly increased, however, the transcript of p53 was not changed. Collectively, these results indicate that the 6 Gy of the IR dose effectively induced premature senescence phenotypes in MCF7 cells.

Selection of marker genes showing correlation according to time after irradiation. To find marker genes reflecting the above-mentioned phenotypes, we selected genes that displayed a significant change (ANOVA p-value within 1\% FDR) and strong correlation (Pearson correlation coefficient $|r|>0.9$ ) with time. From this analysis, 305 genes were found to be altered in a time-dependent manner after exposure to IR and thus were selected as potential stringent markers. Among them, 177 genes showed a positive correlation indicating gradual up-regulation, and 128 genes showed a negative correlation indicating gradual down-regulation after IR exposure. Based on the results of the functional studies, alterations in expression of these genes may explain the phenotypes observed during IR exposure, which suggests that the genes may be canonical markers for IR treatment.

Dynamic modulation of actin structure as a key for the change of cellular shape. Actin protein is the major component of the cytoskeleton, and the rearrangement of actin cytoskeleton plays a pivotal role in dictating cellular shape. Among the 305 markers indentified in the previous section, 14 markers corresponded to genes encoding proteins that are related to the structure of actin (Table I). All of these genes showed positive correlation except Rho GTPase activating protein 19 (ARHGAP19), which encodes a Rho GTPase activation protein, that negatively regulates Rho GTPases by stimulating GTPase activities. In this functional category, there were four more genes encoding GTP binding proteins including Ras homolog gene family, member C (RHOC), RHOQ, RHOU, and Rho family GTPase 1 (RND1). RND1 and RHOU proteins lack intrinsic GTPase activities $(19,20)$, and these two modulators affect the cell shape by regulating the dynamics of the actin cytoskeleton and its reorganization (21). Indeed, the overexpression of $R N D 1$ in fibroblasts inhibits the formation of actin stress fibers, focal adhesions, and membrane ruffles and these effects collectively cause cell rounding (22). The RHOU protein also dissolves stress fibers, disassembles focal adhesions, and induces filopodium formation $(19,20)$. Like the RHOU gene, FYVE, RhoGEF and $P H$ domain containing 3 (FGD3) encode proteins that promote formation of filopodia and induce broad sheet-like protrusions (23). Other GTP binding proteins encoded in the RHOC and RHOQ genes can modulate the actin cytoskeleton (24). Our data showed that the phenotypic morphological change caused by IR exposure was strongly correlated with the collective upregulation of these genes, which are responsible for the dynamics of actin stress fibers and focal adhesion formation.

The remaining genes (Table I) encode proteins that are associated with the actin molecule. Shroom family member 3 (SHROOM3) encodes a protein that colocalizes with F-actin in stress fibers. Supervillin (SVIL) produces a protein that is also known to associate tightly with actin filaments near the plasma membrane. Palladin (PALLD) encodes a protein that is required for the organization of actin cytoskeleton, and the WAS protein family, member 3 (WASF3) encodes an effector molecule that acts as a link between tyrosine kinase receptors and small GTPases which modulate the actin cytoskeleton. There were two genes, Zyxin (ZYX) and Thymosin $\beta 4, X$-linked (TMSB4X) that encode proteins responsible for actin polymerization and depolymerization, respectively. There is a sequential relation between the expression of these two genes; TMSB4X expression up-regulates $Z Y X$ expression (25). Finally, there was Gelsolin (GSN), which encodes a protein that binds directly to the actin monomers and filaments to promote assembly and cleavage of the actin fibers.

Induction of lysosomal metabolic activity accompanying the increase of $S A-\beta-G a l$ positive cells. There were seven gene encoding proteins that were involved in lysosomal metabolism 
Table II. List of genes encoding the enzymes/proteins in lysosomes.

\begin{tabular}{|c|c|c|c|c|c|}
\hline Illumina ID & Symbol & FDR & $\mathrm{r}$ & Role & Gene name \\
\hline ILMN_10502 & SIAE & $9.05 \mathrm{E}-08$ & 0.96 & Saccharide & Sialic acid acetylesterase \\
\hline ILMN_11556 & IDUA & $2.56 \mathrm{E}-06$ & 0.94 & Saccharide & $\alpha$-L-iduronidase \\
\hline ILMN_3081 & PSAP & 2.77E-06 & 0.93 & Saccharide & Prosaposin \\
\hline ILMN_15639 & CTSD & $7.02 \mathrm{E}-06$ & 0.92 & Protein & Cathepsin D \\
\hline ILMN_28173 & GNPTG & 7.99E-06 & 0.92 & Saccharide & $\mathrm{N}$-acetylglucosamine-1-phosphate transferase, $\gamma$ \\
\hline ILMN_23298 & NEU1 & $1.69 \mathrm{E}-07$ & 0.91 & Saccharide & Sialidase 1 (lysosomal sialidase) \\
\hline ILMN_21273 & NAGA & 2.29E-05 & -0.90 & Saccharide & $\alpha-\mathrm{N}$-acetylgalactosaminidase \\
\hline
\end{tabular}

Table III. List of genes encoding proteins involved in the cell cycle and cell proliferation.

\begin{tabular}{|c|c|c|c|c|c|}
\hline Illumina ID & Symbol & FDR & $\mathrm{r}$ & Role & Gene name \\
\hline ILMN_20700 & DUSP1 & $1.15 \mathrm{E}-07$ & 0.95 & Suppression & Dual specificity phosphatase 1 \\
\hline ILMN_19233 & CADM1 & $2.51 \mathrm{E}-05$ & 0.95 & Suppression & Cell adhesion molecule 1 \\
\hline ILMN_27003 & CDK7 & $5.48 \mathrm{E}-06$ & 0.94 & Proliferation & Cyclin-dependent kinase 7 \\
\hline ILMN_18038 & BTG1 & 2.88E-06 & 0.94 & G0/G1 & B-cell translocation gene 1, anti-proliferative \\
\hline ILMN_22260 & SASH1 & $9.55 \mathrm{E}-06$ & 0.91 & Suppression & SAM and SH3 domain containing 1 \\
\hline ILMN_17355 & GADD45A & $1.91 \mathrm{E}-06$ & 0.91 & $\mathrm{G} 2 / \mathrm{M}$ & Growth arrest and DNA-damage-inducible, $\alpha$ \\
\hline ILMN_5648 & PLK2 & $3.65 \mathrm{E}-05$ & 0.91 & Regulation & Polo-like kinase 2 (Drosophila) \\
\hline ILMN_2618 & POLD1 & 4.89E-06 & -0.90 & S & Polymerase (DNA directed), $\delta 1$, catalytic subunit $125 \mathrm{kDa}$ \\
\hline ILMN_27253 & CCNF & $9.76 \mathrm{E}-07$ & -0.90 & $\mathrm{G} 2 / \mathrm{M}$ & Cyclin F \\
\hline ILMN_19730 & E2F2 & $1.44 \mathrm{E}-06$ & -0.90 & $\mathrm{G} 1 / \mathrm{S}$ & E2F transcription factor 2 \\
\hline ILMN_9393 & FOXM1 & $2.07 \mathrm{E}-07$ & -0.91 & G2 & Forkhead box M1 \\
\hline ILMN_12370 & SFN & $9.27 \mathrm{E}-07$ & -0.92 & $\mathrm{G} 2 / \mathrm{M}$ & Stratifin \\
\hline ILMN_14702 & CKS2 & 2.91E-08 & -0.92 & Regulation & CDC28 protein kinase regulatory subunit 2 \\
\hline ILMN_24237 & $\mathrm{HCFC} 1$ & $6.02 \mathrm{E}-07$ & -0.92 & Proliferation & Host cell factor C1 (VP16-accessory protein) \\
\hline ILMN_18431 & POLE & $9.65 \mathrm{E}-09$ & -0.93 & $\mathrm{~S}$ & Polymerase (DNA directed), $\varepsilon$ \\
\hline ILMN_21369 & TIMELESS & $7.57 \mathrm{E}-07$ & -0.93 & $\mathrm{~S}$ & Timeless homolog (Drosophila) \\
\hline
\end{tabular}

(Table II). Aside from one gene, $\alpha$ - $N$-acetyl-galactosaminidase $(N A G A)$, all other genes in this functional category showed a positive correlation. The NAGA gene encodes an enzyme that cleaves the $\alpha-\mathrm{N}$-acetylgalacto-saminyl region from glycoconjugates. Among the remaining genes, four encode enzymes involved in sugar catabolism. $\alpha$-L-iduronidase (IDUA) encodes an enzyme that plays a role in the degradation of glycosaminoglycans. Sialidase 1 (NEU1) encodes an enzyme that cleaves terminal sialic acid residues from glycoproteins and glycolipids. Sialic acid acetylesterase (SIAE) encodes an enzyme that specifically removes the acetyl groups of sialic acids modified by 9-O-acetylation, and the Prosaposin (PSAP) encodes a protein that facilitates the catabolism of glycosphingolipids.

Also, another marker gene, Cathepsin D (CTSD), encoded an acid protease found in lysosomes. Although not directly related to the lysosome, there was a gene encoding a protein that was targeted to the Golgi complex, N-acetylglucosamine-1-phosphate transferase, $\gamma$ subunit (GNPTG). The GNPTG protein is the recognition component of UDP-N- acetylglucosamine:lysosomal-enzyme N-acetylglucosamine1-phosphotransferase complex, which catalyzes the initial step of the synthesis of the mannose-6-phosphate determinant. This modification is required for trafficking of lysosomal hydrolases from the Golgi to lysosome.

Cell cycle arrest and slow-down at $S$ and G2/M phases. There were sixteen marker genes encoding proteins involved in regulating the cell cycle (Table III). Among these genes, several genes encoded proteins that play important roles in the various phases of the cell cycle or display cell-cycle dependent expression patterns. One of these genes, $B$-cell translocation gene 1, anti-proliferative (BTG1) $(\mathrm{r}=0.94)$, which is transcriptionally regulated in a cell cycle-dependent manner, is involved in the G0/G1 phase. BTG1 belongs in the antiproliferative gene family, and its expression is up-regulated in G0 and subsequently down-regulated in G1 (26). Before the cells undergo mitosis (M), the nuclear DNA should be completely replicated in S. Interestingly, our data showed that two major DNA polymerases were down-regulated: 
Table IV. List of genes encoding factors associated with microtubules and spindles.

\begin{tabular}{|c|c|c|c|c|c|}
\hline Illumina ID & Symbol & FDR & $\mathrm{r}$ & Role & Gene name \\
\hline ILMN_29693 & MAP1LC3B & $3.89 \mathrm{E}-06$ & 0.95 & Assembly & Microtubule-associated protein 1 light chain $3 \beta$ \\
\hline ILMN_16399 & TUBB3 & $5.66 \mathrm{E}-05$ & 0.91 & Constituent & Tubulin, $B 3$ \\
\hline ILMN_26809 & GAS8 & 2.61E-05 & 0.90 & Physical interaction & Growth arrest-specific 8 \\
\hline ILMN_12352 & AURKA & $3.66 \mathrm{E}-06$ & -0.90 & Assembly & Aurora kinase A \\
\hline ILMN_11775 & STMN1 & $3.96 \mathrm{E}-07$ & -0.90 & Disassembly & Stathmin 1/oncoprotein 18 \\
\hline ILMN_27641 & TUBB8 & $2.76 \mathrm{E}-07$ & -0.91 & Constituent & Tubulin, $\beta 8$ \\
\hline ILMN_20208 & TPX2 & $6.70 \mathrm{E}-06$ & -0.91 & Spindle & $\begin{array}{l}\text { TPX2, microtubule-associated, homolog } \\
\text { (Xenopus laevis) }\end{array}$ \\
\hline ILMN_418 & TCP1 & $1.89 \mathrm{E}-08$ & -0.91 & Tubulin-folding & T-complex 1 \\
\hline ILMN_137969 & FAM33A & 2.71E-06 & -0.91 & Spindle & Family with sequence similarity 33 , member A \\
\hline ILMN_3509 & TUBB4Q & 2.89E-08 & -0.92 & Constituent & Tubulin, $ß$ polypeptide 4 , member Q \\
\hline ILMN_23841 & GTSE1 & $7.08 \mathrm{E}-06$ & -0.92 & Physical interaction & G-2 and S-phase expressed 1 \\
\hline ILMN_3508 & CALM3 & 4.24E-05 & -0.92 & Spindle & Calmodulin 3 (phosphorylase kinase, $\delta$ ) \\
\hline ILMN_18041 & CENPH & $2.31 \mathrm{E}-07$ & -0.93 & Kinetochore & Centromere protein $\mathrm{H}$ \\
\hline ILMN_4809 & RACGAP1 & $1.59 \mathrm{E}-06$ & -0.93 & Physical interaction & Rac GTPase activating protein 1 \\
\hline ILMN_23465 & $\mathrm{TBCD}$ & $2.42 \mathrm{E}-06$ & -0.95 & Tubulin-folding & Tubulin folding cofactor D \\
\hline ILMN_2123 & $\mathrm{RCC} 2$ & $3.93 \mathrm{E}-06$ & -0.95 & Physical interaction & Regulator of chromosome condensation 2 \\
\hline
\end{tabular}

Polymerase (DNA directed), $\varepsilon$ (POLE) and Polymerase (DNA directed), $\delta 1$, catalytic subunit $125 \mathrm{kDa}(P O L D 1)$. In addition, Timeless homolog (Drosophila) (TIMELESS), which encodes a mediator for DNA replication and the intra-S phase checkpoint (27), also showed a negative correlation. Furthermore, E2F transcription factor 2 (E2F2) encoding a transcription factor that is responsible for the activation of genes required for $\mathrm{G} 1 / \mathrm{S}$, was found to be down-regulated in our data. We also found that the Forkhead box M1 (FOXM1) gene, which encodes a transcription factor responsible for expression of many G2-specific genes, had a negative correlation. Finally, two genes, Growth arrest and DNA damage-inducible, $\alpha$ (GADD45A) and Cyclin F (CCNF), were found to have a positive and a negative correlation, respectively. The GADD45A protein arrests the cell cycle at G2/M, and the CCNF protein is a $\mathrm{G} 2 / \mathrm{M}$ cyclin.

Concerted down-regulation of genes related to microtubulel spindle. There were 15 genes encoding proteins related to microtubules (Table IV). Most of them showed a negative correlation except three genes including Micro-tubuleassociated protein 1 light chain $3 \beta$ (MAP1LC3B), Growth arrest-specific 8 (GAS8), and Tubulin, $\beta 3$ (TUBB3). TUBB3 is a major microtubule constituent, MAP1LC3B is involved in microtubule assembly, and GAS8 is a linker between the microtubules and cytoskeleton. The remaining genes showing a negative correlation were involved in either microtubule structure or spindle organization/biogenesis.

Among the down-regulated genes, six genes were related to microtubule structure including the genes encoding two major microtubule constituents, such as Tubulin, $\beta$ polypeptide 4, member $Q$ (TUBB4Q) and Tubulin, $\beta 8$ (TUBB8). The T-complex 1 (TCP1) gene, that encodes a molecular chaperone responsible for folding tubulin proteins, was also down-regulated. The Regulator of chromosome condensation 2 (RCC2) and G2/S-phase expressed 1 (GTSE1) genes encode proteins known either to interact or to colocalize with microtubules. The Stathmin1/oncoprotein 18 (STMN1) gene, that encodes a protein responsible for disassembly of microtubules, was also found to be down-regulated. Stathmin overexpression is known to associate with highly proliferative breast cancer cells (28), and the suppression of stathmin expression shows tumor-suppression function in breast cancer cell lines that contain a mutation in p53 (29).

Although all the above-mentioned genes could be directly or indirectly related to the spindle structure, seven genes are known to be definitively involved in spindle organization and biogenesis. Both Aurora kinase A (AURKA) and TPX2, microtubule-associated, homolog (Xenopus laevis) (TPX2) encode proteins that locate to the spindle microtubules and the spindle poles. The proteins encoded in these genes work sequentially; TPX2 first finds the spindle microtubules and then recruits AURKA (30). The Calmodulin 3 (phosphorylase kinase, $\delta$ ) (CALM3) gene encodes a protein that is also known to localize to spindle poles during mitosis. A recent result shows that one of our markers, Tubulin folding cofactor $D$ $(T B C D)$, which encodes a tubulin-folding protein, plays an important role in the organization of the mitotic spindle (31). The Rac GTPase activating protein 1 (RACGAP1) gene encodes for a protein that accumulates to the mitotic spindle during metaphase and condenses in the midbody during cytokinesis (32). Family with sequence similarity 33, member $A$ (FAM33A) (Ska2), which encodes for a component of the spindle and kinetochore (KT)-associated complex, is required for timely anaphase onset (33). Overall the negative correlation of all these markers responsible for spindle organization and biogenesis strongly reflects the active mitotic suppression in the radiation-treated MCF7 cells. 
Table V. Genes responsible for RNA processing and translation.

\begin{tabular}{|c|c|c|c|c|c|}
\hline Illumina ID & Symbol & FDR & $\mathrm{r}$ & Role & Gene name \\
\hline ILMN_10407 & SFRS14 & 7.77E-06 & 0.93 & Splicing process & Splicing factor, arginine/serine-rich 14 \\
\hline ILMN_24701 & PABPC1 & 2.95E-08 & 0.92 & Translation & Poly(A) binding protein, cytoplasmic 1 \\
\hline ILMN_139052 & EIF4A2 & $1.96 \mathrm{E}-06$ & 0.92 & Translation & Eukaryotic translation initiation factor $4 \mathrm{~A}$, isoform 2 \\
\hline ILMN_15031 & RPS29 & $2.80 \mathrm{E}-07$ & 0.91 & Protein synthesis & Ribosomal protein S29 \\
\hline ILMN_3458 & YBX2 & $1.63 \mathrm{E}-05$ & -0.90 & Translation & $\mathrm{Y}$ box binding protein 2 \\
\hline ILMN_12476 & MRPL41 & $8.58 \mathrm{E}-07$ & -0.90 & Protein synthesis & Mitochondrial ribosomal protein L41 \\
\hline ILMN_1567 & NXT2 & $1.28 \mathrm{E}-06$ & -0.91 & Nuclear export & Nuclear transport factor 2-like export factor 2 \\
\hline ILMN_16446 & EIF4EBP2 & 4.53E-06 & -0.91 & Translation & $\begin{array}{l}\text { Eukaryotic translation initiation factor } 4 \mathrm{E} \text { binding } \\
\text { protein } 2\end{array}$ \\
\hline ILMN_20407 & PTBP1 & 4.53E-07 & -0.91 & Splicing process & Polypyrimidine tract binding protein 1 \\
\hline ILMN_19199 & GLE1 & $1.47 \mathrm{E}-05$ & -0.91 & Nuclear export & GLE1 RNA export mediator homolog (yeast) \\
\hline ILMN_15237 & IPO8 & 4.31E-07 & -0.91 & Nuclear import & Importin 8 \\
\hline ILMN_8699 & SNRPC & $3.83 \mathrm{E}-05$ & -0.91 & Splicing process & Small nuclear ribonucleoprotein polypeptide C \\
\hline ILMN_18679 & MRPL38 & $1.05 \mathrm{E}-05$ & -0.92 & Protein synthesis & Mitochondrial ribosomal protein L38 \\
\hline ILMN_25262 & METAP1 & $1.18 \mathrm{E}-05$ & -0.92 & Protein synthesis & Methionyl aminopeptidase 1 \\
\hline ILMN_26447 & HNRPA2B1 & 3.97E-07 & -0.92 & Splicing process & Heterogeneous nuclear ribonucleoprotein A2/B1 \\
\hline ILMN_17736 & UTP18 & 7.39E-07 & -0.92 & rRNA processing & $\begin{array}{l}\text { UTP18, small subunit (SSU) processome component, } \\
\text { homolog (yeast) }\end{array}$ \\
\hline ILMN_24933 & MRPL48 & $3.41 \mathrm{E}-06$ & -0.93 & Protein synthesis & Mitochondrial ribosomal protein L48 \\
\hline ILMN_14120 & MRPL18 & $2.45 \mathrm{E}-06$ & -0.93 & Protein synthesis & Mitochondrial ribosomal protein L18 \\
\hline ILMN_7818 & DARS2 & $2.13 \mathrm{E}-06$ & -0.93 & Protein synthesis & Aspartyl-tRNA synthetase 2 , mitochondrial \\
\hline ILMN_7614 & HEATR1 & $2.06 \mathrm{E}-08$ & -0.94 & rRNA processing & HEAT repeat containing 1 \\
\hline ILMN_4967 & MRPL11 & $5.05 \mathrm{E}-08$ & -0.95 & Protein synthesis & Mitochondrial ribosomal protein L11 \\
\hline
\end{tabular}

Down-regulation of activities in transcription and translation. There were 21 genes encoding proteins that are involved in RNA processing and translation (Table V). Most of these genes showed a negative correlation. Among the negatively correlated genes, the following were involved in mRNA splicing: Polypyrimidine tract binding protein 1 (PTBP1), Small nuclear ribonucleoprotein polypeptide C (SNRPC), and Heterogeneous nuclear ribonucleoprotein A2/B1 (HNRPA2B1). Additionally, there were two genes related to mature mRNA transportation, Nuclear transport factor 2-like export factor 2 (NXT2) and GLE1 RNA export mediator homolog (yeast) (GLE1). The products of these genes play sequential roles in nuclear transport. The down-regulation of these genes indicates the IR exposure resulted in the suppression of overall post-transcriptional activity.

We also found two genes that directly affect mRNA structure, which in turn alters translation initiation, and one gene that affects ribosomal recognition of mRNA. A Eukaryotic translation initiation factor 4A (EIF4A) isoform, EIF4A2 encodes a protein that has RNA helicase activity (34). The EIF4A protein melts the secondary structure of the 5 ' untranslated region of RNA to facilitate binding of the $40 \mathrm{~S}$ ribosomal subunit. The $\operatorname{Poly}(A)$ binding protein, cytoplasmic 1 ( $P A B P C 1)$ gene encodes a protein that binds to the poly(A) tail and regulates translation initiation. Both EIF4A2 and PABPC1 were positively correlated with IR treatment. In addition, the EIF4EBP 2 gene, which is involved in translation initiation, was negatively correlated with IR exposure. This protein sequesters EIF4E and prevents it from binding with EIF4F, which ultimately prevents translation initiation (35).

We also observed several negatively-correlated genes that are directly involved in protein synthesis. They included two genes involving in ribosomal RNA processing, UTP18, small subunit (SSU) processome component (UTP18) and HEAT repeat containing 1 (HEATR 1$)$. There were several genes encoding mitochondrial ribosomal protein large subunits (MRPLs) including MRPL41, MRPL38, MRPL48, MRPL18, and MRPL11. Importin 8 (IPO8) gene, which showed a negative correlation, was also included in this functional category, and its encoded protein is responsible for the nuclear import of nuclear-targeted proteins. Overall, most of these changes result in the down-regulation of activities in post-transcription and translation except the specific upregulation at the translation initiation stage.

\section{Discussion}

Cellular senescence historically has been described in normal human cells undergoing a finite number of divisions before permanent cell cycle arrest (36). However, it is now recognized that the senescence phenotype can be also achieved in cancer cells through a variety of different treatments (37). Recent studies have reported that induction of premature senescence may be a promising method to treat cancer $(4,38)$. We previously demonstrated that IR effectively induced 
premature senescence in human breast carcinoma MCF7 cells. To identify markers for detecting senescent cancer cells, we performed comparative proteomic analysis during senescence induction in response to IR. Using this approach, we found that cathepsin $\mathrm{D}(\mathrm{CD})$ and eukaryotic translation elongation factor $1 \alpha 1$ (eEF1A1) hold promise as markers for the detection of cellular senescence (39). However, there is still lack of knowledge regarding the mechanism of IR-induced senescence.

To identify genes specifically associated with the premature senescence phenotype induced by IR exposure, we examined changes in cellular properties and global gene expression in the breast cancer cell line MCF7 during 4 days after irradiation. The radiation changed the overall morphology of MCF7 cells to a senescence-like morphology and increased SA-B-Gal activity. The cell cycle profile indicated that the cells were arrested at G1 and the S phase disappeared. The lack of proliferative activity in irradiated cells was confirmed using a colony forming assay. The radiation changed the global pattern of gene expression as well as the morphology and phenotype. The 305 markers that displayed both significant changes after radiation and strong correlations with time were identified. Since these marker genes also could indicate characteristics of long-term radiation effects, they might be relevant to understand mechanism of remedy. Through functional analysis, these markers were divided into a few specific categories including actin cytoskeleton structure, lysosome activity, cell cycle, spindle structure and protein synthesis. Altered expression of these markers was consistent with the changes in the morphological characteristics of the cells observed after IR exposure.

Most of the markers associated with the actin cytoskeleton structure were up-regulated. The actin cytoskeleton is a key determinant of cell shape. The gradual increase in expression of these genes might be responsible for the gradual morphological changes in cell shape after irradiation. We also found that expression of 7 genes encoding proteins for lysosomal activity was altered. Most of these genes showed a positive correlation and were in good agreement with the SA- $\beta-$ Gal assay results. Lysosomal $\beta$-galactosidase activity has been shown to increase in senescent cells due to increased lysosome content and activity (40). Moreover, NEU1, which was positively correlated with IR exposure, is detected in a lysosome-targeted multiprotein complex with $\beta$-galactosidase and protective protein/cathepsin A (PPCA) (41), and causes the desialylation of both platelet-derived growth factor (PDGF) and insulin-like growth factor 1 (IGF-1) receptors and diminishes the intracellular signals induced by their mitogenic ligands (42).

Among the markers associated with the regulation of the cell cycle, 16 genes were directly related to the cell cycle process. These genes were responsible for transitioning the cell cycle from G0 to G1, and from S to G2/M. Regardless whether these genes were positively or negatively correlated, any of these changes could strongly arrest the cell cycle progression by altering cell cycle checkpoints. We observed concerted down-regulation of genes related to the microtubule and spindle structures. A total of 15 genes were identified in this functional category, and seven genes were directly involved in the spindle structure. These genes are involved in centrosomal regulation during mitotic division. Some of the proteins encoded in these genes could serve as the main microtubule organizing center (MTOC) of the animal cell as well as a regulator of cell-cycle progression. Its aberrant structure or inactivation of MTOC leads to cell cycle arrest, mitotic catastrophe or apoptotic cell death (43). The markers for the cell cycle and the spindle structure categories strongly suggest the transcriptional end stage of the cell cycle arrest, specifically $\mathrm{M}$ on the transcriptional level. We also found that overall protein synthesis might be down-regulated. There was consistent suppression of genes for the mitochondrial ribosomal large subunits and for the processing enzymes of cytosolic rRNAs. Previous studies have shown a strong decrease in ATP production in senescent human fibroblasts, together with increased mitochondrial ROS production $(44,45)$. However, some genes that are involved in translation initiation, showed positive correlations.

Many studies have examined gene expression profiles in replicative senescence in normal human cells $(46,47)$. It has been also reported that IR exposure induces changes in the gene expression profiles of cancer cells, which has simply been denoted as IR-response genes $(7,32,48,49)$. Since no study has previously examined and characterized the gene expression profile in senescent cancer cells after IR exposure in a time-dependent manner, we defined our selected markers as senescence-associated genes, which were highly consistent with the senescent phenotype induced by IR exposure in cancer cells. We believe the results of this study could provide a better understanding of the molecular mechanism of senescence progression induced by IR treatment.

\section{Acknowledgements}

This study was supported by the National Nuclear Technology Program from The Ministry of Education, Science, and Technology of Korea (for J.S. Lee), by the Korea Science and Engineering Foundation (KOSEF) grant funded by the Korea government (MEST) (No.2009-0084110) (for C.K. Lee), and by the Shared Research Equipment Assistance Program by the Korea Basic Science Institute, MEST (for S.H. Leem).

\section{References}

1. Chu K, Teele N, Dewey MW, Albright N and Dewey WC: Computerized video time lapse study of cell cycle delay and arrest, mitotic catastrophe, apoptosis and clonogenic survival in irradiated 14-3-3sigma and CDKN1A (p21) knockout cell lines. Radiat Res 162: 270-286, 2004.

2. Kis E, Szatmari T, Keszei M, et al: Microarray analysis of radiation response genes in primary human fibroblasts. Int $\mathbf{J}$ Radiat Oncol Biol Phys 66: 1506-1514, 2006.

3. Feldser DM and Greider CW: Short telomeres limit tumor progression in vivo by inducing senescence. Cancer Cell 11: 461-469, 2007.

4. Gewirtz DA, Holt SE and Elmore LW: Accelerated senescence: an emerging role in tumor cell response to chemotherapy and radiation. Biochem Pharmacol 76: 947-957, 2008.

5. Cosme-Blanco W, Shen MF, Lazar AJ, et al: Telomere dysfunction suppresses spontaneous tumorigenesis in vivo by initiating p53-dependent cellular senescence. EMBO Rep 8: 497-503, 2007.

6. Campisi J and d'Adda di Fagagna F: Cellular senescence: when bad things happen to good cells. Nat Rev Mol Cell Biol 8: 729-740, 2007. 
7. Collado M, Blasco MA and Serrano M: Cellular senescence in cancer and aging. Cell 130: 223-233, 2007.

8. Dimri GP, Lee X, Basile G, et al: A biomarker that identifies senescent human cells in culture and in aging skin in vivo. Proc Natl Acad Sci USA 92: 9363-9367, 1995.

9. Goletz TJ, Smith JR and Pereira-Smith OM: Molecular genetic approaches to the study of cellular senescence. Cold Spring Harb Symp Quant Biol 59: 59-66, 1994.

10. Campisi J: Suppressing cancer: the importance of being senescent Science 309: 886-887, 2005.

11. Mooi WJ and Peeper DS: Oncogene-induced cell senescencehalting on the road to cancer. N Engl J Med 355: 1037-1046, 2006.

12. Narita M and Lowe SW: Senescence comes of age. Nat Med 11: 920-922, 2005

13. Ly DH, Lockhart DJ, Lerner RA and Schultz PG: Mitotic misregulation and human aging. Science 287: 2486-2492, 2000

14. Shelton DN, Chang E, Whittier PS, Choi D and Funk WD: Microarray analysis of replicative senescence. Curr Biol 9: 939-945, 1999

15. Zhang H, Pan KH and Cohen SN: Senescence-specific gene expression fingerprints reveal cell-type-dependent physical clustering of up-regulated chromosomal loci. Proc Natl Acad Sci USA 100: 3251-3256, 2003.

16. Fridman AL and Tainsky MA: Critical pathways in cellular senescence and immortalization revealed by gene expression profiling. Oncogene 27: 5975-5987, 2008.

17. Coppe JP, Patil CK, Rodier F, et al: Senescence-associated secretory phenotypes reveal cell-non-autonomous functions of oncogenic RAS and the p53 tumor suppressor. PLoS Biol 6: 2853-2868, 2008

18. Cichowski K and Hahn WC: Unexpected pieces to the senescence puzzle. Cell 133: 958-961, 2008.

19. Saras J, Wollberg P and Aspenstrom P: Wrch1 is a GTPasedeficient Cdc42-like protein with unusual binding characteristics and cellular effects. Exp Cell Res 299: 356-369, 2004.

20. Shutes A, Berzat AC, Cox AD and Der CJ: Atypical mechanism of regulation of the Wrch-1 Rho family small GTPase. Curr Biol 14: 2052-2056, 2004

21. Van Aelst L and D'Souza-Schorey C: Rho GTPases and signaling networks. Genes Dev 11: 2295-2322, 1997.

22. Nobes CD, Lauritzen I, Mattei MG, Paris S, Hall A and Chardin P: A new member of the Rho family, Rnd1, promotes disassembly of actin filament structures and loss of cell adhesion. J Cell Biol 141: 187-197, 1998.

23. Hayakawa M, Matsushima M, Hagiwara H, et al: Novel insights into FGD3, a putative GEF for Cdc42, that undergoes SCF (FWD1/beta-TrCP)-mediated proteasomal degradation analogous to that of its homologue FGD1 but regulates cell morphology and motility differently from FGD1. Genes Cells 13: 329-342, 2008.

24. Chunqiu Hou J and Pessin JE: Lipid Raft targeting of the TC10 amino terminal domain is responsible for disruption of adipocyte cortical actin. Mol Biol Cell 14: 3578-3591, 2003.

25. Moon HS, Even-Ram S, Kleinman HK and Cha HJ: Zyxin is upregulated in the nucleus by thymosin beta4 in SiHa cells. Exp Cell Res 312: 3425-3431, 2006.

26. Rouault JP, Rimokh R, Tessa C, et al: BTG1, a member of a new family of antiproliferative genes. EMBO J 11: 1663-1670, 1992

27. Unsal-Kacmaz K, Mullen TE, Kaufmann WK and Sancar A: Coupling of human circadian and cell cycles by the timeless protein. Mol Cell Biol 25: 3109-3116, 2005

28. Curmi PA, Nogues C, Lachkar S, et al: Overexpression of stathmin in breast carcinomas points out to highly proliferative tumours. Br J Cancer 82: 142-150, 2000

29. Alli E, Yang JM and Hait WN: Silencing of stathmin induces tumor-suppressor function in breast cancer cell lines harboring mutant p53. Oncogene 26: 1003-1012, 2007.

30. Kufer TA, Sillje HH, Korner R, Gruss OJ, Meraldi P and Nigg EA: Human TPX2 is required for targeting Aurora-A kinase to the spindle. J Cell Biol 158: 617-623, 2002.
31. Cunningham LA and Kahn RA: Cofactor D functions as a centrosomal protein and is required for the recruitment of the gamma-tubulin ring complex at centrosomes and organization of the mitotic spindle. J Biol Chem 283: 7155-7165, 2008.

32. Hirose K, Kawashima T, Iwamoto I, Nosaka T and Kitamura T: MgcRacGAP is involved in cytokinesis through associating with mitotic spindle and midbody. J Biol Chem 276: 5821-5828, 2001.

33. Hanisch A, Sillje HH and Nigg EA: Timely anaphase onset requires a novel spindle and kinetochore complex comprising Ska1 and Ska2. EMBO J 25: 5504-5515, 2006.

34. Rogers GW Jr, Richter NJ and Merrick WC: Biochemical and kinetic characterization of the RNA helicase activity of eukaryotic initiation factor 4A. J Biol Chem 274: 12236-12244, 1999.

35. Sonenberg $\mathrm{N}$ and Dever TE: Eukaryotic translation initiation factors and regulators. Curr Opin Struct Biol 13: 56-63, 2003.

36. Hayflick L and Moorhead PS: The serial cultivation of human diploid cell strains. Exp Cell Res 25: 585-621, 1961.

37. Hampton T: Scientists study 'senescence' in cancer: therapies aim to hold cells in a non-dividing state. JAMA 298: 2357-2358, 2007.

38. Roninson IB, Broude EV and Chang BD: If not apoptosis, then what? Treatment-induced senescence and mitotic catastrophe in tumor cells. Drug Resist Updat 4: 303-313, 2001.

39. Byun HO, Han NK, Lee HJ, et al: Cathepsin D and eukaryotic translation elongation factor 1 as promising markers of cellular senescence. Cancer Res 69: 4638-4647, 2009.

40. Kurz DJ, Decary S, Hong Y and Erusalimsky JD: Senescenceassociated (beta)-galactosidase reflects an increase in lysosomal mass during replicative ageing of human endothelial cells. J Cell Sci 113 ( Pt 20): 3613-3622, 2000.

41. Lukong KE, Elsliger MA, Chang Y, et al: Characterization of the sialidase molecular defects in sialidosis patients suggests the structural organization of the lysosomal multienzyme complex. Hum Mol Genet 9: 1075-1085, 2000

42. Hinek A, Bodnaruk TD, Bunda S, Wang Y and Liu K: Neuraminidase-1, a subunit of the cell surface elastin receptor, desialylates and functionally inactivates adjacent receptors interacting with the mitogenic growth factors PDGF-BB and IGF-2. Am J Pathol 173: 1042-1056, 2008.

43. Loffler H, Lukas J, Bartek J and Kramer A: Structure meets function, centrosomes, genome maintenance and the DNA damage response. Exp Cell Res 312: 2633-2640, 2006.

44. Hutter E, Renner K, Pfister G, Stockl P, Jansen-Durr P and Gnaiger E: Senescence-associated changes in respiration and oxidative phosphorylation in primary human fibroblasts. Biochem J 380: 919-928, 2004.

45. Zwerschke W, Mazurek S, Stockl P, Hutter E, Eigenbrodt E and Jansen-Durr P: Metabolic analysis of senescent human fibroblasts reveals a role for AMP in cellular senescence. Biochem J 376: 403-411, 2003.

46. Semov A, Marcotte R, Semova N, Ye X and Wang E: Microarray analysis of E-box binding-related gene expression in young and replicatively senescent human fibroblasts. Anal Biochem 302: 38-51, 2002.

47. Kang MK, Kameta A, Shin KH, Baluda MA, Kim HR and Park NH: Senescence-associated genes in normal human oral keratinocytes. Exp Cell Res 287: 272-281, 2003.

48. Akerman GS, Rosenzweig BA, Domon OE, et al: Alterations in gene expression profiles and the DNA-damage response in ionizing radiation-exposed TK6 cells. Environ Mol Mutagen 45: 188-205, 2005

49. Nagtegaal ID, Gaspar CG, Peltenburg LT, et al: Radiation induces different changes in expression profiles of normal rectal tissue compared with rectal carcinoma. Virchows Arch 446: $127-135,2005$. 